\title{
Frequency and magnetic field dependence of the skin depth in Co- rich soft magnetic microwires
}

\author{
Arcady Zhukov ${ }^{1,2,3}$, Ahmed Talaat ${ }^{1,2}$, Mihail Ipatov ${ }^{1,2}$, Alexandr Granovsky ${ }^{4}$ and Valentina \\ Zhukova $^{1,2}$ \\ ${ }^{1}$ Dpto. de Fís. Mater., UPV/EHU San Sebastián 20009, Spain \\ ${ }^{2}$ Dpto. de Física Aplicada, EUPDS, UPV/EHU, 20018, San Sebastian, Spain \\ ${ }^{3}$ IKERBASQUE, Basque Foundation for Science, 48011 Bilbao, Spain \\ ${ }^{4}$ Faculty of Physics, Lomonosov Moscow State University, 11991 Moscow, Russian Federation \\ *corresponding author, E-mail: arkadi . joukov@ehu.es
}

\begin{abstract}
Tuneable metamaterials consisting of thin ferromagnetic wires exhibiting magnetoimpedance effect present tuneability of the effective permittivity by a weak magnetic field or a mechanical stress. Magnetoimpedance effect is related to magnetic field dependence of the skin depth of magnetic conductor. We studied giant magnetoimpedance (GMI) effect in magnetically soft amorphous Co-rich microwires in the extended frequency range. From obtained experimentally dependences of GMI ratio on magnetic field and different frequencies we estimated the penetration depth and its dependence on applied magnetic field and frequency.
\end{abstract}

\section{Introduction}

Studies of tuneable metamaterials utilising thin ferromagnetic wires exhibiting magnetoimpedance effect (MI) attracted considerable attention along the last few years $[1,2]$. These metamaterials contain arrays of metallic wires and present strong dispersion of the dielectric function in the $\mathrm{GHz}$ frequency range.

Magnetic wires presenting MI effect are the key factors for design of these tuneable metamaterials. Studies of magnetic wires have attracted considerable attention of researchers and engineers along many years $[3,4]$. Perfectly cylindrical symmetry is quite favorable for achievement of high MI effect [3,4]. Consequently giant magnetoimpedance (GMI) effect has been discovered [3] and later intensively studied [4- 7] in different families of soft magnetic wires.

The origin of the aforementioned GMI effect is satisfactory explained considering the change in the penetration depth of the alternating current flowing through the magnetically soft conductor caused by the applied static magnetic field. Recently we modified the experimental facility that allowed us to extend the frequency range and measure GMI effect at GHZ frequencies [5].
In soft magnetic amorphous wires subjected to an external magnetic field the GMI is in the range of $100 \%$ even at frequencies of few $\mathrm{GHz}$ [4-6].

Recently we reported on high MI effect in Co and Fe-rich microwires [5-7].As it is well-known, the penetration depth, $\delta$, depends on the current frequency. For observation of high MI effect the penetration depth must be smaller than the magnetic wires diameter.

Consequently we present our recent studies on the penetration depth of the alternating current flowing through the magnetically soft conductor caused by the applied static magnetic field in thin amorphous wires.

\section{Experimental details}

We studied various Co-rich $\left(\mathrm{Co}_{67} \mathrm{Fe}_{3.85} \mathrm{Ni}_{1.45} \mathrm{~B}_{11.5} \mathrm{Si}_{14.5} \mathrm{Mo}_{1.7}\right.$ and $\mathrm{Co}_{67.71} \mathrm{Fe}_{4.28} \mathrm{Ni}_{1.57} \mathrm{Si}_{11.24} \mathrm{~B}_{12.4} \mathrm{Mo}_{1.25} \mathrm{C}_{1.55}$ ) with different metallic nucleus diameter, $d$, and total microwire diameter, $D$, produced by the modified Taylor-Ulitovsky method described elsewhere

We have measured the magnetic field dependence of impedance, $\mathrm{Z}$, and GMI ratio, $\Delta \mathrm{Z} / \mathrm{Z}$, for various Co-rich microwires $[5,6]$.

We used a specially designed micro-strip sample holder described elsewhere [5]. The sample holder was placed inside a sufficiently long solenoid that creates a homogeneous magnetic field, $H$. The sample impedance, $Z$, was measured using a vector network analyzer from reflection coefficient $S_{11}$. defined as:

The magneto impedance ratio, $\Delta Z / Z$, has been

$$
\Delta Z / Z=\left[Z(H)-Z\left(H_{\max }\right)\right] \cdot 100 / Z\left(H_{\max }\right)
$$

An axial $D C$-field with a maximum value $H_{\max }$ up to 8 $\mathrm{kA} / \mathrm{m}$ was supplied by magnetizing coils.

The frequency range for the diagonal impedance component has been measured from $1 \mathrm{MHz}$ up to $7 \mathrm{GHz}$. 


\section{Experimental results and discussion}

As described above the diameter reduction must be associated with the increasing of the optimal MI frequency range: a tradeoff between dimension and frequency is required in order to obtain a maximum $\mathrm{MI}$ effect $[5,8]$.

Consequently we measured GMI effect at different frequencies.

Co-rich microwires present linear hysteresis loops and high GMI effect in as-prepared state (Fig 1a) and a maximum on frequency dependence of $\Delta Z / Z_{\max }$ can be observed (Fig. 1b).

For $\mathrm{Co}_{67} \mathrm{Fe}_{3.85} \mathrm{Ni}_{1.45} \mathrm{~B}_{11.5} \mathrm{Si}_{14.5} \mathrm{Mo}_{1.7}$ microwire with $d=8.5$ $\mu \mathrm{m}$ the optimum frequency, $f_{o} \approx 100 \mathrm{MHz}$, while for
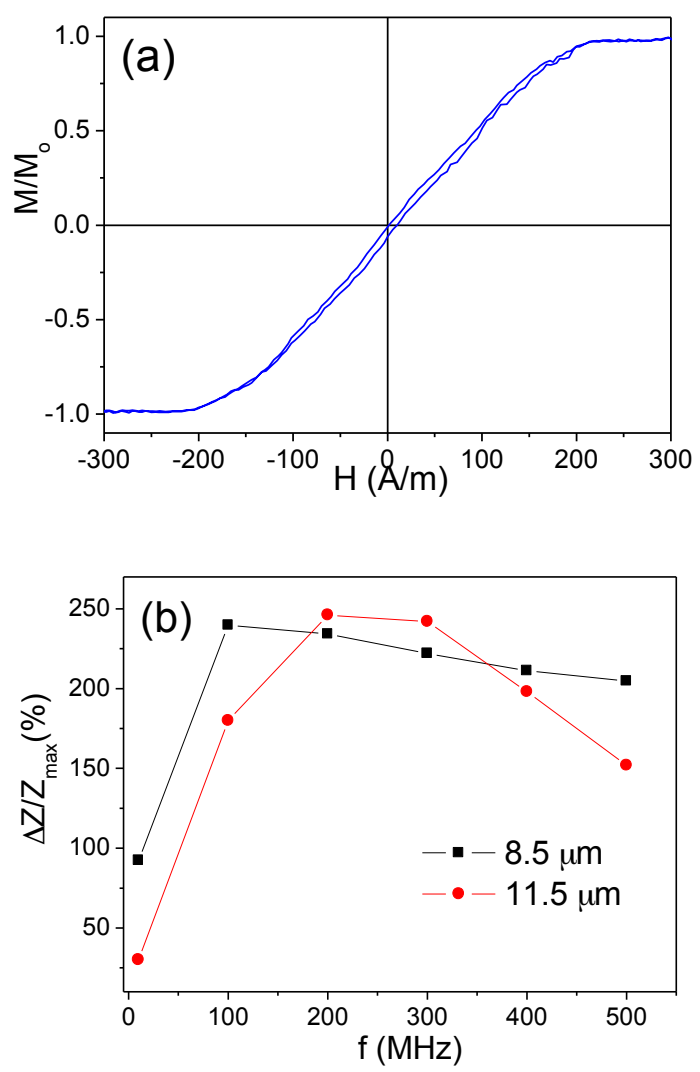

Fig. 1. Hysteresis loop(a) and frequency dependence of $\Delta Z / Z_{\max }$ (b) measured in $\mathrm{Co}_{67} \mathrm{Fe}_{3.85} \mathrm{Ni}_{1.45} \mathrm{~B}_{11.5} \mathrm{Si}_{14.5} \mathrm{Mo}_{1.7}$ microwires with different d.

$\mathrm{Co}_{67} \mathrm{Fe}_{3.85} \mathrm{Ni}_{1.45} \mathrm{~B}_{11.5} \mathrm{Si}_{14.5} \mathrm{Mo}_{1.7}$ microwire with $d=11.5 \mu \mathrm{m}, f_{o}$ $\approx 200 \mathrm{MHz}$.

From $\Delta Z / Z(H)$ dependences it is possible to estimate the penetration depth at different frequencies using the model previously described in ref. (9) considering that the changes in the real component of the impedance are related to changes in the effective area where the AC-current flows as a consequence of the skin-effect. In this model the penetration depth, $\delta$, as a function of the ratio $R_{D C} / R_{A C}\left(R_{D C}\right.$ is the DC-resistance of the wire, and $\mathrm{R}_{\mathrm{AC}}$ is the real component of the impedance, , can be expressed as:

$\delta=r\left[1-(1-R D C / R A C)^{1 / 2}\right]$,

where $r$ is the wire radius.

Consequently we measured $\Delta Z / Z(H)$ dependences for various Co-rich microwires and tried to estimate the $\delta(H)$ dependences.

Obtained $\delta(H)$ dependences demonstrate that at high frequencies the minimum penetration depth of $\mathrm{Co}_{67} \mathrm{Fe}_{3.85} \mathrm{Ni}_{1.45} \mathrm{~B}_{11.5} \mathrm{Si}_{14.5} \mathrm{Mo}_{1.7}$ microwire is about $1.5 \mu \mathrm{m}$ (Fig.2).

Similalrly for $\mathrm{Co}_{67.71} \mathrm{Fe}_{4.28} \mathrm{Ni}_{1.57} \mathrm{Si}_{11.24} \mathrm{~B}_{12.4} \mathrm{Mo}_{1.25} \mathrm{C}_{1.55}$ microwirew $(d \approx 10 \mu \mathrm{m})$ GMI effect is observed even at $\mathrm{GHz}$ frequenciwes (Fig. 3a). For the case of
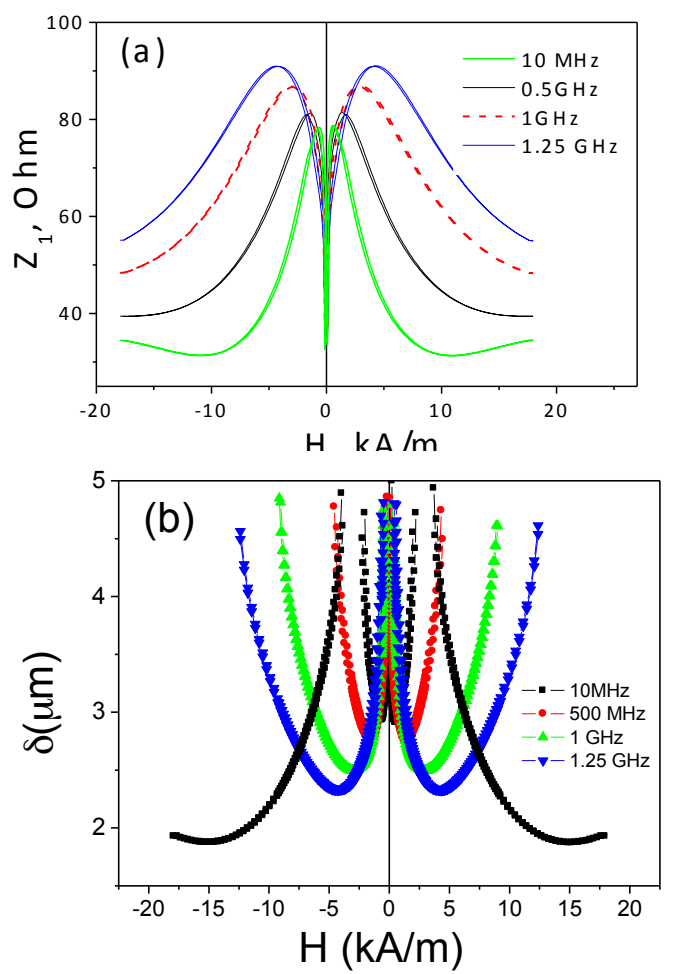

Fig.2. $\mathrm{Z}(\mathrm{H})$ dependence (a) and calculated $\delta(H)$ dependences (b) of $\mathrm{Co}_{67} \mathrm{Fe}_{3.85} \mathrm{Ni}_{1.45} \mathrm{~B}_{11.5} \mathrm{Si}_{14.5} \mathrm{Mo}_{1.7}$ microwires estimated for different frequencies.

$\mathrm{Co}_{67.71} \mathrm{Fe}_{4.28} \mathrm{Ni}_{1.57} \mathrm{Si}_{11.24} \mathrm{~B}_{12.4} \mathrm{Mo}_{1.25} \mathrm{C}_{1.55}$ microwire the minimum calculated penetration depth is below $0.5 \mu \mathrm{m}$ for high frequencies (Fig.3b). From obtained $\delta(H)$ dependences we evaluated $\delta$-values and dependence of minimum $\delta$ values on frequency (see Fig.3c). As can be appreciated from Fig.3c minimum $\delta$-values, $\delta_{\text {min }}$, decrease with increasing the frequency and at $2 \mathrm{GHz} \delta_{\min }, \approx 0.33 \mu \mathrm{m}$ (Fig.3c).

Consequently we observed non-monotonic $\delta(H)$ dependences that must be attributed to GMI effect (Figs2b and $3 \mathrm{~b}$ ) and monotonic decreasing of the $\delta_{\min }$ with increasing the frequency.

The penetration depth, $\delta$, is given by: 

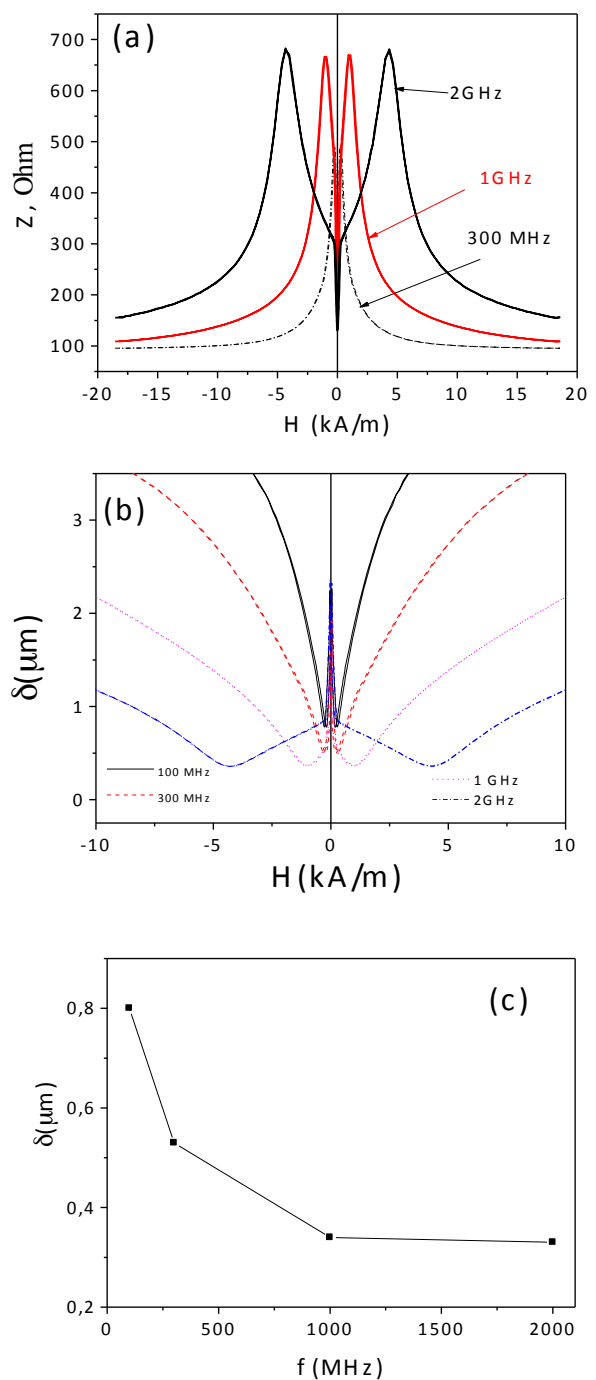

Fig.3. $\mathrm{Z}(\mathrm{H})$ dependence (a), calculated $\delta(H)$ dependences (b) and $\delta_{\min }(\mathrm{f})$ (c) of $\mathrm{Co}_{67.71} \mathrm{Fe}_{4.28} \mathrm{Ni}_{1.57} \mathrm{Si}_{11.24} \mathrm{~B}_{12.4} \mathrm{Mo}_{1.25} \mathrm{C}_{1.55}$ microwires $(d=10 \mu \mathrm{m}, D=13.8 \mu \mathrm{m})$ at different frequencies.

$$
\delta=\left(\pi \sigma \mu_{\phi} f\right)^{-1 / 2}
$$

where $\sigma$ is the electrical conductivity, $f$ the frequency of the current along the sample, and $\mu_{\phi}$ the circular magnetic permeability $[3,8]$. The $d c$ applied magnetic field introduces significant changes in the circular permeability, $\mu_{\phi}$. Therefore, the penetration depth also changes giving rise to non-monotonic $\delta(H)$ dependences [3,9]. On the other hand the decreasing of $\delta_{\min }$ with increasing the frequency must be attributed to the classical skin effect $[3,10]$. The electric current flows mainly at the "skin" of the conductor, between the outer surface and a level called the skin depth. The skin effect causes the effective resistance of the conductor to increase with increasing the frequency.
Consequently obtained minimum penetration depth for studied Co-rich microwires is few times smaller than the microwires diameter.

\section{Conclusions}

We measured the GMI effect in Co-rich magnetic wires. From obtained experimentally dependences of GMI ratio on magnetic field and different frequencies we estimated the skin depth and its dependence on applied magnetic field and frequency.

\section{Acknowledgements}

This work was supported by Spanish MINECO under MAT2013-47231-C2-1-P and the Russian Science Foundation under the 16-19-10490 grant. Technical and human support provided by SGIker (UPV/EHU, MICINN, $\mathrm{GV} / \mathrm{EJ}, \mathrm{ERDF}$ and ESF) is gratefully acknowledged.

\section{References}

[1] D.P. Makhnovskiy, L.V. Panina, C. Garcia, A.P. Zhukov, and J. Gonzalez, Experimental demonstration of tunable scattering spectra at microwave frequencies in composite media containing $\mathrm{CoFeCrSiB}$ glass-coated amorphous ferromagnetic wires and comparison with theory, Phys. Rev. B, Vol. 74 No. 6: 2006.

[2] M. Ipatov, V. Zhukova, A. Zhukov and L. V. Panina, Microwave Metamaterials Containing Magnetically Soft Microwires, Advances in Science and Technology, 75: 224-229, 2010.

[3] L.V. Panina and K. Mohri, Magneto-impedance effect in amorphous wires," Appl. Phys. Lett., 65: 1189-1191, 1994.

[4] J. M. Blanco, A. Zhukov and J. Gonzalez, Effect of tensile and torsion on GMI effect in amorphous wire, $J$. Magn. Magn. Mat. 196-197: 377-379, 1999.

[5] A. Zhukov, A. Talaat, M. Ipatov and V. Zhukova, Tailoring the high-frequency giant magnetoimpedance effect of amorphous Co-rich microwires, IEEE Magn. Lett., 6: 2500104, 2015.

[6] A. Zhukov, M. Ipatov and V. Zhukova, Advances in Giant Magnetoimpedance of Materials, Handbook of Magnetic Materials, ed. K.H.J. Buschow, 24: chapter 2, 139-236, 2015

[7] D.C. Jiles, Recent advances and future directions in magnetic materials. Acta Mater. 51: 5907-5939, 2003

[8] D. Ménard, M. Britel, P. Ciureanu and A. Yelon, Giant magnetoimpedance in a cylindrical conductor, J. Appl. Phys. 84: 2805-2814, 1998.

[9] H. Lachowicz, K.L. García, M. Kuzminski, A. Zhukov and M. Vázquez, Skin effect and circumferential permeability in micro-wires utilized in GMI-sensors, Sensors and Actuators A, 119: 384-389, 2005.

[10] Hayt, William, Engineering Electromagnetics (4th ed.), McGraw-Hill, ISBN 0-07-027395-2 , 1981. 